\title{
TONICIDAD: ¿UNA PROPIEDAD DE LAS SOLUCIONES Y/O DE LAS CÉLULAS? APRENDIZAJE SUSTENTABLE DEL TRANSPORTE DE AGUA
}

\section{Tonicity: a Property of Solutions and / or Cells? Sustainable Learning of Water Transport}

\section{Tonicidade: uma propriedade de soluções e / ou das células? Aprendizagem sustentável do transporte de água}

\author{
Nazira Píriz* \\ María Noel López Larrama** \\ Jerónimo Tucci*** \\ Joselín Cantero ${ }^{\star \star \star \star}$ \\ Virginia Mallarini $^{\star \star * \star *}$
}

\section{Resumen}

El estudio de procesos biológicos en un laboratorio y con protocolos simplificados puede introducir o fortalecer errores conceptuales. Este es el caso del transporte de agua, cuando se enseña como ósmosis. El presente trabajo describe una experiencia que atiende dicha problemática. Introduce el concepto de transporte de agua diferenciándolo del de ósmosis, plantea dificultades en su enseñanza, señala conceptos inclusores erróneos frecuentes, fundamenta por qué protocolos de uso habitual fortalecen dichos conceptos erróneos y relata experiencias sustentadas en el Modelo de aprendizaje cognitivo consciente sustentable. Dichas experiencias proponen protocolos ampliados que contribuyen a la introducción de conceptos sostén relevantes, favoreciendo un aprendizaje sustentable del transporte de agua.

Palabras clave: transporte de agua; ósmosis; permeabilidad; aprendizaje sustentable; conceptos sostén

\footnotetext{
* Consejo de Formación en Educación y Consejo Directivo Central de la ANEp, Uruguay. Correo electrónico: nazirapiriz@gmail.com.

** Consejo de Formación en Educación, ANEp. Uruguay. Correo electrónico: marianoelopez@gmail.com

***Consejo de Formación en Educación, ANEp, Uruguay. Correo electrónico: jeronimotucci@gmail.com

**** Consejo de Formación en Educación, ANEp, Uruguay. Correo electrónico: joselincantero@gmail.com

***** Consejo de Formación en Educación, ANEP, Uruguay. Correo electrónico: vir.malla28@gmail.com
} 


\section{Abstract}

The study of biological processes in a laboratory and with simplified protocols can introduce or strengthen conceptual errors. This is the case of water transport, when it is taught as osmosis. This work describes an experience that addresses this problem. It introduces the concept of water transport differentiating it from that of osmosis, it poses difficulties in its teaching, it points out frequent erroneous misconceptions, it bases why habitual protocols strengthen these misconceptions and recounts experiences sustained in the Sustainable Cognitive Conscious Learning Model. These experiences propose extended protocols that contribute to the introduction of relevant support concepts, favoring a sustainable learning of water transport.

Keywords: water transport; osmosis; permeability; sustainable learning; support concepts

\section{Resumo}

O estudo de processos biológicos em laboratório e com protocolos simplificados pode introduzir ou fortalecer erros conceituais. É o caso do transporte aquático, quando ensinado como osmose. Este artigo descreve uma experiência que aborda esse problema. Introduz o conceito de transporte de água que o diferencia da osmose, apresenta dificuldades em seu ensino, aponta equívocos inclusivos frequentes, comprova porque os protocolos comumente usados fortalecem esses equívocos e narra experiências com base no modelo de aprendizado cognitivo consciente sustentável. Essas experiências propõem protocolos ampliados que contribuem para a introdução de conceitos relevantes de suporte, favorecendo um aprendizado sustentável do transporte de água.

Palavras chave: transporte aquático; osmose; permeabilidade; aprendizado sustentável; conceitos de apoio 


\section{Introducción}

En los seres vivos se verifican regularidades, en ocasiones denominadas leyes o principios, descritas en las ciencias naturales en general. No obstante, los seres vivos presentan características propias que los diferencian de entidades no vivas. Es así que sistemas físico-químicos artificiales y preparados biológicos en situaciones simplificadas suelen constituir modelos parciales y limitados, y no siempre adecuados para la explicación de procesos biológicos. A modo de ejemplo, esto se da cuando, con el objetivo de estudiar el equilibrio osmótico en los seres vivos, se utilizan sistemas conformados por compartimentos separados por membranas que se comportan como semipermeables para los solutos elegidos. Esta inadecuación se debe a que, en condiciones fisiológicas, en los seres vivos, el pasaje de agua se da por un proceso denominado transporte de agua, que difiere del conocido con el nombre de ósmosis. La diferencia entre ambos no es una mera discrepancia de términos o denominaciones, sino que implica variantes conceptuales de relevancia en la enseñanza de las ciencias biológicas. Por este motivo, nos parece oportuno atender a esta diferencia, mediante estrategias didácticas que permitan evidenciarla. Este trabajo presenta actividades experimentales que, desde la perspectiva del modelo de aprendizaje cognitivo consciente sustentable (Galagovsky, 2004a y 2004b), permiten que quien aprende diferencie de forma consciente los conceptos de concentración, osmolaridad y osmolaridad efectiva, y comprenda además la relevancia de la permeabilidad específica de las membranas biológicas a diversos solutos y su influencia en el transporte de agua, lo anterior con un aprendizaje sustentable de dicho proceso y del concepto de tonicidad.

\section{¿En qué se diferencian la ósmosis y el transporte de agua?}

Se define como ósmosis el pasaje de agua a través de membranas semipermeables. Por definición, una membrana semipermeable es aquella que solo permite el pasaje de solvente (agua) pero no de solutos. Los organismos vivos están organizados en diferentes compartimentos, que pueden (de forma simplificada) considerarse soluciones de solutos, cuyo solvente es el agua. Dichos compartimentos están separados por membranas biológicas (celulares, de organelos), siempre permeables al agua, y con permeabilidad selectiva a diversos solutos. Teniendo en cuenta además que los seres vivos son sistemas abiertos, resulta claro que intercambian materia y energía con su entorno, no limitándose al agua. Se desprende de esto que las membranas biológicas no son semipermeables, sino que tienen permeabilidad selectiva.
En condiciones fisiológicas, lo habitual es que el pasaje de agua y de solutos se dé de forma conjunta, por lo que el proceso de ósmosis queda limitado a situaciones experimentales particulares. Además, el pasaje de solutos a través de membranas biológicas modifica las osmolaridades de los medios que dichas membranas separan, y varía también el pasaje de agua (Cingolani y Houssay, 2006; Frumento, 1995; Glaser, 2003; Latorre, Barneo, Bezanilla y Llinas, 1996; Píriz Giménez, 2016; Píriz y López, 2011). Esto trae como consecuencia que información limitada a diferencias en la osmolaridad de las soluciones sea insuficiente para predecir cambios en el volumen de las células, pudiendo encontrar resultados inesperados con determinados protocolos. La osmolaridad efectiva, determinada por la permeabilidad de la membrana a los solutos en juego, explica tales resultados. Resulta más adecuado, entonces, en la enseñanza de las Ciencias Biológicas, hablar de "transporte de agua" en los seres vivos, y de "ósmosis" solo en determinadas situaciones experimentales (pero no fisiológicas), o en sistemas no-biológicos.

\section{¿Qué dificultades aparecen con frecuencia en la enseñanza del transporte de agua en los seres vivos?}

La enseñanza del proceso de ósmosis y su vinculación con los seres vivos ha sido preocupación de diversos autores (Aiziczon y Cudmani, 2012; Aragüés y Sáez, 2014; Díaz y Abuín, 1988). Entre 2008 y 2018 (López, Tucci y Píriz Giménez, 2016; Píriz y López, 2011; Píriz Giménez, Tucci y Cantero, 2018), nuestro grupo de trabajo ha relevado datos de pruebas diagnósticas, participaciones orales y pruebas escritas en cursos de profesorado en Ciencias Biológicas y en cursos de profundización para egresados, que han permitido identificar dificultades que se reiteran en distintas generaciones. Estas se presentan a continuación:

- Atribuir el término concentración a las soluciones en lugar de a los solutos, y, por ende, utilizar expresiones tales como soluciones concentradas para soluciones de varios solutos (como los compartimentos que separan las membranas biológicas).

- Utilizar el término concentración para cantidad, desconociendo que el primero de ellos se refiere a la distribución espacial del soluto y que, por ende, el volumen de la solución está implícito en dicha magnitud.

- Utilizar el término concentración como osmolaridad, desconociendo la jerarquía de la disociación de solutos en solución y que, por tanto, si el soluto cambia, a una misma concentración le pueden corresponder diferentes osmolaridades. 
- Considerar las diferencias de concentraciones de soluciones como el factor determinante del pasaje de agua a través de membranas biológicas.

- Desconocer que el pasaje de solutos a través de las membranas modifica secundariamente el pasaje de agua, y que el coeficiente de reflexión permite corregir este factor.

- Utilizar el término ósmosis para todo pasaje de agua a través de membranas biológicas.

- Categorizar las membranas biológicas como semipermeables y en ocasiones como sinónimo de permeabilidad selectiva.

- Desconocer las expresiones osmolaridad efectiva y tonicidad, así como su significado.

\section{Cuando los protocolos prácticos fortalecen conceptos inclusores erróneos}

El modelo de aprendizaje cognitivo consciente sustentable plantea la necesidad de que quien aprende se haga consciente de sus errores conceptuales, como parte fundamental de un proceso de aprendizaje. El modelo diferencia entre conceptos sostén y conceptos inclusores, en vista de que estos últimos pueden ser incorrectos, de modo que su vinculación puede dar coherencia y lógica a un aprendizaje significativo, pero erróneo. Asimismo, plantea la relevancia de considerar en la enseñanza el logro de diversos conceptos sostén que puedan vincularse y conformar una red que perdure en el tiempo (Galagovsky, 2004a y 2004b).

Entendemos que en la problemática presentada los protocolos prácticos utilizados habitualmente tanto en la Educación Media como en la Educación superior (Burbano, Caetano de Almeida, Otero y Álvarez, 2017; Camarena Rosales, 2017; Chávez Torres, Medina Barriga, Sánchez Suárezy Mendoza Olivares, 2013; Contreras Ramírez, 2017; Corominas, 2010; Gómez e Illana, 2007; Ramos, 2016; Torre Casares, 2005) refuerzan las dificultades enumeradas. Dichos protocolos contribuyen a vincular conceptos inclusores inadecuados que enumeramos a continuación, y que dada su coherencia fortalecen un aprendizaje significativo pero incorrecto.

Los conceptos inclusores erróneos fortalecidos en protocolos habituales son los siguientes:

- Las membranas biológicas son semipermeables.

- El pasaje de agua en los seres vivos constituye un proceso de ósmosis.

- El pasaje de agua en los seres vivos se da por diferencias de concentración de solutos.

\section{¿Por qué dichos protocolos contribuyen a un aprendizaje erróneo del transporte de agua?}

El motivo radica en que dichos protocolos utilizan en todos los casos un mismo soluto (habitualmente $\mathrm{NaCl}$ ). Esto trae consigo las siguientes consecuencias:

- Impide incorporar la variable de disociación de solutos, puesto que un mismo soluto se disocia de una única manera, lo que no permite diferenciar concentración de osmolaridad.

- Al usar un mismo soluto tampoco se puede considerar variantes en la permeabilidad de las membranas celulares, por tanto no es posible diferenciar osmolaridad de osmolaridad efectiva.

\section{¿Cómo deconstruir esos conceptos inclusores erróneos y construir conceptos sostén?}

En atención a las debilidades descritas en los protocolos prácticos que se usan habitualmente en la enseñanza de las ciencias biológicas, elegimos a modo de estrategia crear protocolos prácticos que incorporen:

- Solutos diversos que se diferencien en su disociación y en la permeabilidad con respecto a las membranas biológicas.

- Diferentes células, con membranas cuya permeabilidad a los solutos propuestos también difiera.

Una experiencia exitosa para el aprendizaje sustentable del transporte de agua

Presentamos una propuesta experimental aplicada en cursos de profesorado en Ciencias Biológicas de dos instituciones (Instituto de Profesores Artigas y Modalidad Semipresencial de Profesorado), en 2017 y 2018. La propuesta se desarrolló con un total de 120 estudiantes de 18 años o más, en grupos de no más de 10 estudiantes.

La estrategia elegida fue utilizar información provista por diversas fuentes de internet, que explique el pasaje de agua a través de membranas biológicas. Con base en dicha información se orientó a los estudiantes para formular una primera hipótesis de trabajo y, a partir de ella, proponer un protocolo para corroborarla. 
Asimismo, se brindó a los estudiantes la siguiente información:

La tonicidad de una solución es una propiedad que se relaciona con el efecto que tiene dicha solución en el volumen celular. Dado que el agua ocupa un gran porcentaje del volumen en las células, sus cambios se explican por salida o ingreso de agua a las células. Se llama solución hipotónica a aquella que produce ingreso de agua a las células; solución hipertónica aquella que produce salida de agua de las células; y solución isotónica la que no produce cambios en el volumen celular.

Se plantea a los estudiantes que dicha información es meramente descriptiva y no explica las causas en los cambios de volumen celular, a lo que se intentará llegar con las actividades experimentales.

A continuación, se sintetiza el desarrollo de la propuesta, que abarcó 3 días de trabajo en el laboratorio.

\section{Día 1}

La información tomada de internet de la que se partió fue la siguiente:

¿Por qué sale agua de las células?... La ósmosis es el movimiento neto de agua a través de una membrana semipermeable desde una zona de baja concentración de solutos hacia otra de mayor concentración. (Khan Academy).

Con base en esta información, planteamos el siguiente razonamiento: Si el pasaje de agua a través de una membrana biológica depende únicamente de diferencias en la concentración de solutos entre los medios separados por dicha membrana (sin importar el tipo de soluto y el tipo de membrana), entonces si colocamos células en contacto con soluciones de igual concentración de solutos, tendremos necesariamente los mismos resultados en las células. Es decir, que todas las soluciones utilizadas se comportarán como hipotónicas, como hipertónicas o como isotónicas, independientemente del soluto y las células utilizadas. A partir de esta idea, los estudiantes acordaron lo siguiente:

- Hipótesis 1. Soluciones de diferentes solutos a igual concentración, tendrán los mismos efectos en preparados con células.

A continuación, se les preguntó qué protocolo seguirían para probar si dicha hipótesis es correcta. Sin dificultades, propusieron utilizar dos soluciones de diferentes solutos a igual concentración y someter un mismo tipo celular a ambas.
Se ofreció a los estudiantes sangre ovina como fuente de eritrocitos y las siguientes soluciones: solución 0,3 $\mathrm{M}$ de $\mathrm{NaCl}$ y solución $0,3 \mathrm{M}$ de sacarosa. Los estudiantes consideraron que los materiales ofrecidos cumplían los requisitos y pasaron a elaborar los preparados y observarlos (figura 1).

Los resultados obtenidos al microscopio óptico con aumento 40X fueron los siguientes:
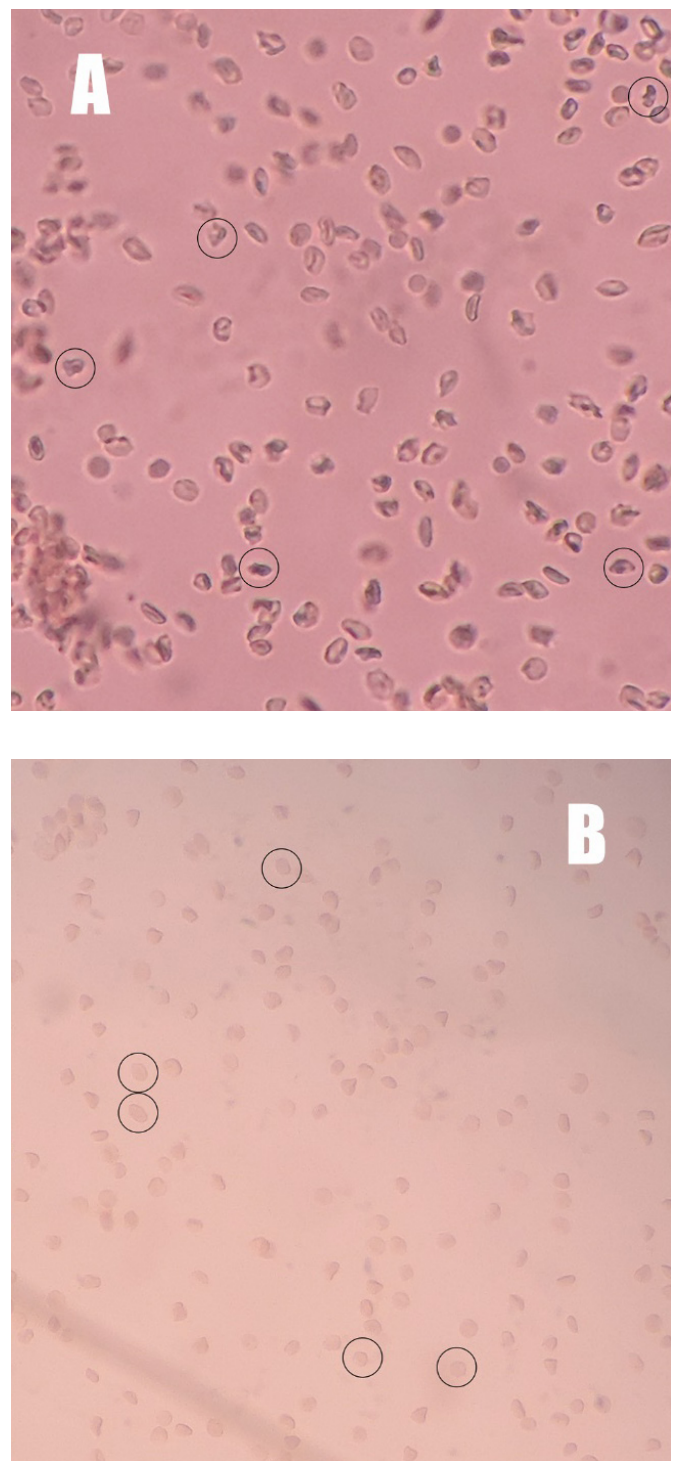

Figura 1. Eritrocitos sometidos a soluciones $A$. $\mathrm{NaCl}$ 0,3 M, y $B$. sacarosa 0,3 M. Copyrigth 2019 por María Noel López.

Los estudiantes corroboraron que mientras la solución $0,3 \mathrm{M}$ de $\mathrm{NaCl}$ determinó la crenación de eritrocitos, la solución 0,3 M de sacarosa, no. En esta última se puede observar su conocida forma bicóncava. Es decir, comprobaron que, a pesar de que usaron soluciones de igual 
concentración, la de $\mathrm{NaCl}$ resultó hipertónica, en tanto que la de sacarosa resultó isotónica. Se concluyó entonces que dos soluciones a igual concentración de diferente soluto en un mismo tipo celular pueden no determinar los mismos efectos en el pasaje de agua a través de las membranas celulares, así que rechazaron la primera hipótesis. Para la siguiente clase (día 2), a partir de una revisión bibliográfica, los alumnos debían buscar una explicación para los resultados encontrados y formular una nueva hipótesis.

\section{Día 2}

Los estudiantes encontraron que la diferencia entre la sacarosa y el $\mathrm{NaCl}$ radica en que mientras el primer soluto no se disocia, el segundo sí. De este modo, si bien la concentración de ambos solutos en solución es la misma, no lo es su osmolaridad (que se calcula como el producto entre la molaridad y el número de partículas en que se disocia el soluto). De esta manera, mientras la solución $0,3 \mathrm{M}$ de $\mathrm{NaCl}$ tiene una osmolaridad de 0,6 osmolar (dado que el $\mathrm{NaCl}$ se disocia en dos partículas: $\mathrm{Na}+\mathrm{y} \mathrm{Cl}-$ ), la solución $0,3 \mathrm{M}$ de sacarosa tiene una osmolaridad de 0,3 osmolar, dado que la sacarosa no se disocia en agua. También encontraron que la osmolaridad es una magnitud más adecuada que la concentración para explicar el pasaje de agua a través de membranas.

Los estudiantes diferenciaron entonces concentración de osmolaridad. Así derribaron un primer concepto inclusor y formularon una segunda hipótesis de trabajo:

- Hipótesis 2. Soluciones de diferentes solutos a igual osmolaridad tendrán los mismos efectos en preparados con células.

Una vez más, los estudiantes debieron proponer un nuevo protocolo para verificar si dicha hipótesis era correcta. Sin dificultades, propusieron utilizar dos soluciones de diferentes solutos a igual osmolaridad y someter un mismo tipo celular a ambas.

Se ofreció a los estudiantes sangre ovina como fuente de eritrocitos y las siguientes soluciones: solución $0,3 \mathrm{M}$ de sacarosa y solución 0,3 M de urea.

Los estudiantes corroboraron que ni la sacarosa ni la urea se disocian, por lo que ambas soluciones presentan la misma osmolaridad (0,3 osmolar). Consideraron que los materiales ofrecidos cumplían los requisitos y pasaron a elaborar los preparados y observarlos (figura 2).
Los resultados obtenidos al microscopio óptico con aumento 40X fueron los siguientes:
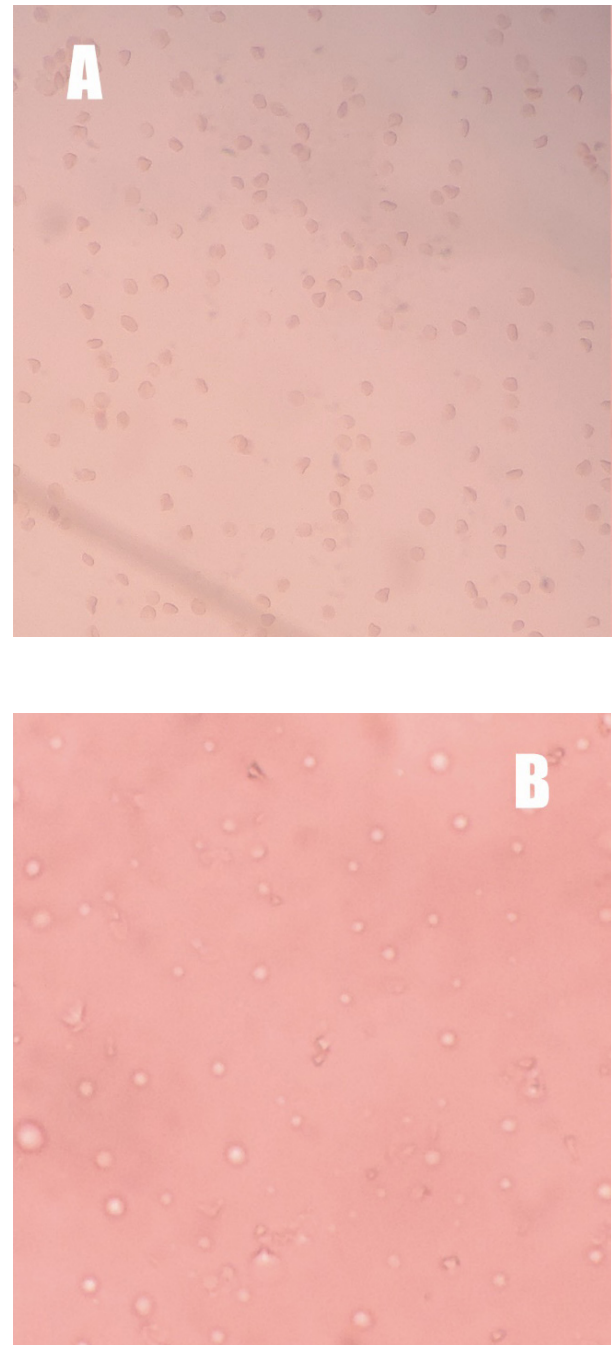

Figura 2. Eritrocitos sometidos a soluciones $A$. sacarosa $0,3 \mathrm{M}$, y $B$. urea 0,3 M. Copyrigth 2019 por Nazira Píriz.

Los estudiantes corroboraron que mientras en la solución $0,3 \mathrm{M}$ de sacarosa los eritrocitos se veían redondeados, en la solución 0,3M de urea, no se veían eritrocitos.

Adicionalmente, la observación macroscópica de los tubos de ensayo también difería. Mientras que el tubo con sangre y sacarosa se veía opaco, el tubo con sangre y urea se veía translúcido (figura 3). Esto último se debe a la ausencia de eritrocitos por lisis.

Los resultados permitieron interpretar que mientras la solución 0,3 osmolar de sacarosa se comportó como isotónica, la solución 0,3 osmolar de urea se comportó como hipotónica. 


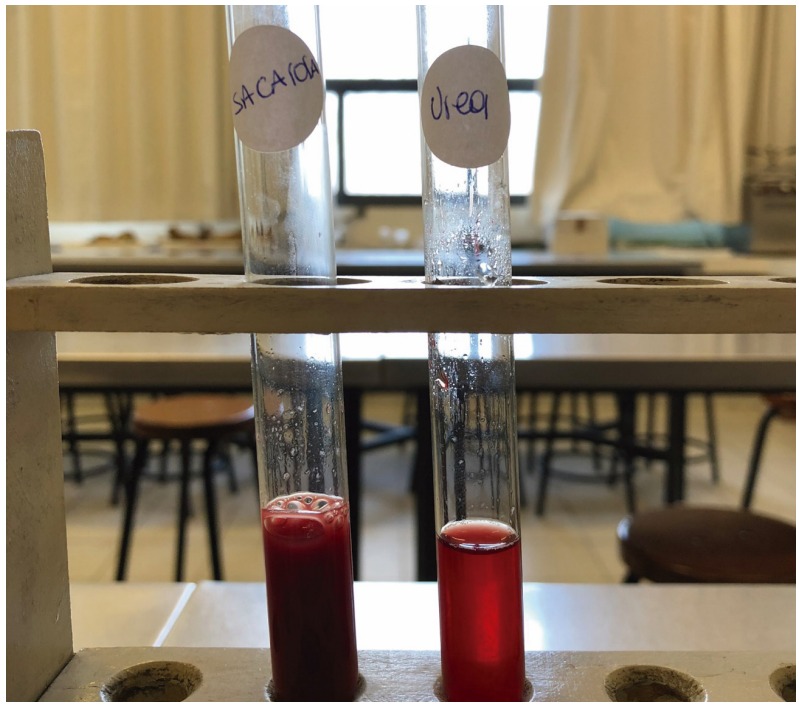

Figura 3. Preparados macroscópicos de eritrocitos en soluciones de sacarosa y urea, ambas a 0,3 M. Copyrigth 2019 por Jerónimo Tucci.

Los estudiantes concluyeron que los efectos en un mismo tipo celular de dos soluciones a igual osmolaridad de diferente soluto pueden no ser los mismos, por lo que rechazaron también la segunda hipótesis.

Para la siguiente clase se les solicitó que buscaran información que permitiera explicar los resultados encontrados y formular una nueva hipótesis.

\section{Día 3}

Los estudiantes encontraron en la bibliografía que la diferencia entre los solutos utilizados radica en el coeficiente de reflexión, un parámetro relacionado con la permeabilidad de las membranas a los solutos, que puede diferir. Explicaron que dado que la urea es permeable a la membrana de eritrocitos ovinos, y por ser un soluto neutro, su alta concentración fuera de las células determina que dicho soluto ingrese a ellas por gradiente químico. El ingreso de urea aumenta la osmolaridad intracelular de los eritrocitos y determina secundariamente el ingreso de agua. Debido a que este fenómeno se repite, el ingreso mantenido de agua a las células determina su lisis. De esta manera se explica la ausencia de eritrocitos en los campos observados al microscopio, así como el carácter translúcido del tubo con el preparado con la solución isosmolar de urea. En este caso, una solución con la misma osmolaridad que el interior de los eritrocitos (y por ende isosmolar) se comportó como hipotónica. Esto demuestra que la osmolaridad y la tonicidad no solo son propiedades diferentes, sino que no son "propias" de las soluciones, y que la tonicidad depende del tipo celular con que estas se encuentren en contacto.
Se introdujo de esta manera el concepto de osmolaridad efectiva, asociado al de tonicidad, y los estudiantes propusieron una tercera hipótesis como definitiva:

- Hipótesis definitiva. Soluciones de diferentes solutos a igual osmolaridad efectiva tendrán los mismos efectos en preparados con células.

La comprobación de esta última hipótesis requeriría la utilización de solutos con igual coeficiente de reflexión para un mismo tipo celular, lo que es prácticamente imposible. Por este motivo se dio por finalizada la actividad práctica, aceptando que con los conocimientos actuales, dicha hipótesis no puede rechazarse.

En esta oportunidad los estudiantes diferenciaron: osmolaridad de osmolaridad efectiva; membrana semipermeable de membrana con permeabilidad selectiva; y ósmosis de transporte de agua. Además, lograron vincular osmolaridad efectiva con tonicidad, y concluyeron que esta última no es una propiedad exclusiva de las soluciones, sino que depende también del tipo celular. Los estudiantes también lograron incorporar en forma consciente dichos términos como nuevos conceptos sostén y derribaron todos los conceptos inclusores erróneos presentados.

\section{Síntesis y conclusiones de la experiencia relatada}

Es de destacar la participación activa e interesada de los estudiantes tanto en la formulación de hipótesis como en la propuesta y aplicación de los protocolos así como en la explicación de los resultados. Esta participación junto con la posibilidad de experimentary vivenciar permitieron que se dieran cuenta de sus conceptos erróneos y construyeran nuevos conceptos sostén, de acuerdo con el modelo de aprendizaje cognitivo consciente sustentable.

Los estudiantes recibieron con entusiasmo la propuesta, y los resultados sorpresivos despertaron mayor motivación para buscar información y lograr explicarlos, por lo que la experiencia se valoró muy positivamente.

\section{Cuando esperamos plasmólisis y encontramos turgencia}

El protocolo presentado fue ampliado con variantes en diferentes cursos, incorporando células de planta, de modo que se vincularan los términos plasmólisis y turgencia con el transporte de agua. 
Debemos considerar que en el caso de células de planta, dependiendo del preparado utilizado, diferirá el indicador del volumen de la vacuola, y por ende el del volumen celular. En células con vacuolas con antocianinas (pigmento violáceo), la intensidad del color y su disposición en la célula ayudan a interpretar si hubo salida o ingreso de agua en ella. En células sin pigmentos pero con alta densidad de cloroplastos, como la Egeria densa, su ubicación permitirá concluir sobre este aspecto.

A continuación presentamos resultados experimentales de observaciones microscópicas en distintos preparados de células de planta (Egeria densa y Allium cepa), en las que se utilizaron las siguientes soluciones en forma comparativa (figura 4):

- Solución saturada de $\mathrm{NaCl}(\mathrm{A})$

- Solución saturada de urea (B)

- Solución saturada de sacarosa (C)

- Solución saturada de glucosa (D)

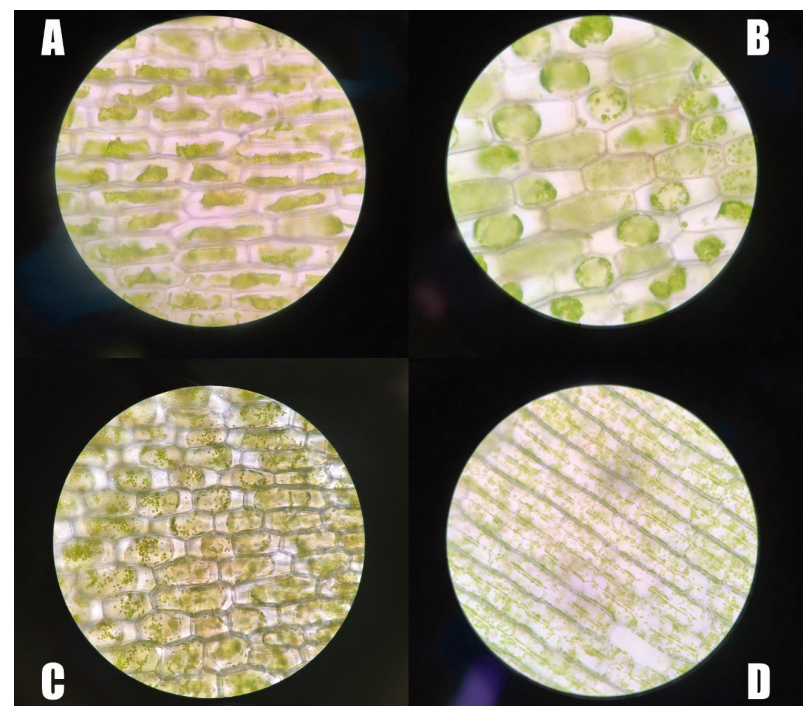

Figura 4. Preparados de Egeria densa (nombre vulgar: Elodea) en soluciones saturadas de $A$. $\mathrm{NaCl}, B$. urea, $C$. sacarosa y $D$. glucosa. Copyrigth 2019 por Virginia Mallarini y Joselín Cantero.

Las observaciones permiten interpretar que en Egeria densa las soluciones saturadas de $\mathrm{NaCl}$, urea y sacarosa se comportaron como hipertónicas (dada la distribución central de los cloroplastos que sugieren una reducción por salida de agua del volumen de la vacuola), en tanto que la solución saturada de glucosa se comportó como hipotónica (dada la distribución periférica de los cloroplastos que sugieren un incremento por entrada de agua en el volumen de la vacuola) (véase la figura 5).

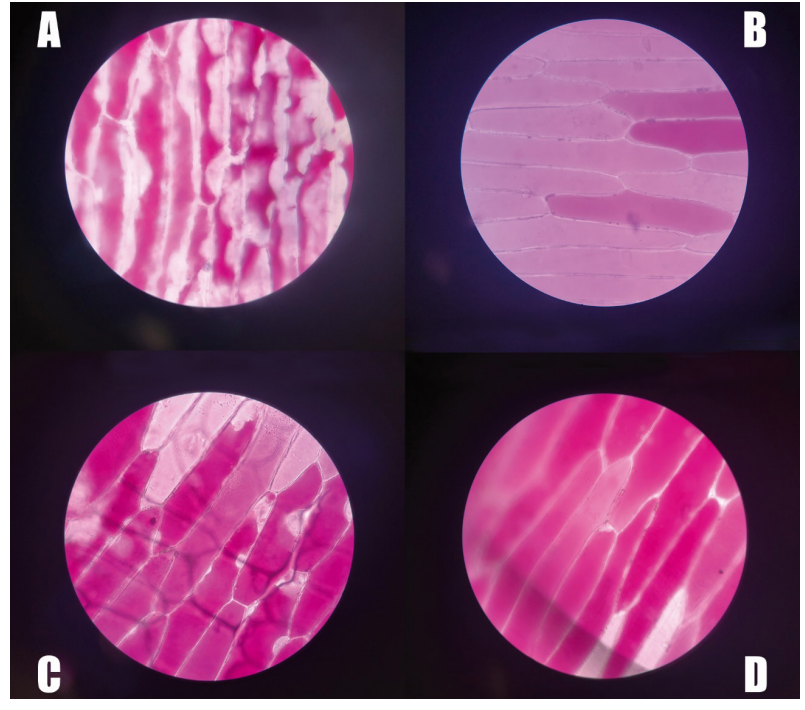

Figura 5. Preparados de Allium cepa var. Rosum (nombre vulgar: cebolla colorada), en soluciones saturadas de $A$. $\mathrm{NaCl}, B$. urea, C. sacarosa y D. glucosa. Copyright 2019 por María Noel López y Nazira Píriz.

Es posible interpretar que en Aliumm cepa la solución saturada de $\mathrm{NaCl}$ se comportó como hipertónica, en tanto que las de urea, sacarosa y glucosa se comportaron como hipotónicas. La distribución de la coloración de la vacuola permite inferir salida de agua de las células en el primer caso e ingreso en los siguientes.

\section{Conclusiones}

Los resultados muestran que si nos limitamos al uso de protocolos habituales (Burbano et ál., 2017; Camarena Rosales, 2017; Chávez et ál., 2013; Contreras Ramírez, 2017; Corominas, 2010; Gómez et ál., 2007; Ramos, 2016; Torre Casares et ál., 2005) que utilizan un único soluto que además no permea en las membranas utilizadas, encontraríamos en todos los preparados que una solución a alta concentración de soluto se comporta como hipertónica, en tanto que una solución a baja concentración de soluto se comporta como hipotónica. Estos resultados suelen adelantarse en dichos protocolos y favorecen una generalización inadecuada, lo cual refuerza el concepto ampliamente difundido de que el pasaje de agua depende de la concentración del soluto. Por este motivo planteamos que la utilización de soluciones de un único soluto contribuye a reforzar las dificultades enumeradas en este artículo y que han resultado de investigaciones previas (Píriz et ál., 2018; López et ál., 2016; Píriz y López, 2011).

La incorporación de varios solutos en preparados de células diferentes permite evidenciar en el laboratorio que mientras las soluciones saturadas de urea y sacarosa resultan hipertónicas para el preparado en Egeria densa, 
se comportan como hipotónicas en Allium cepa. De este modo, se ilustra con claridad que la tonicidad es una propiedad que no depende únicamente de las soluciones sino también de las células con las que se ponen en contacto. Diversidad de células implica también diversidad de permeabilidades y efectos.

Diferencias en la expresión de proteínas transportadoras en distintas membranas pueden determinar que ciertas células sean permeables a algunos solutos pero no a otros. El pasaje de solutos por gradiente químico hacia el interior celular (dado que tanto la urea, la sacarosa y la glucosa son solutos neutros) aumenta secundariamente la osmolaridad intracelular determinando el ingreso de agua. Cabe recordar que, como solutos hidrosolubles, la sacarosa y la urea no atraviesan las membranas biológicas por difusión simple sino a través de proteínas transportadoras específicas. Dado que la expresión de proteínas transportadoras difiere en distintas células, encontramos que un mismo soluto puede ser osmóticamente activo para cierta célula, pero no para otra. Es decir que un soluto que no permea a través de determinada membrana celular (y que tendrá un coeficiente de reflexión igual a 1), no atravesará dicha membrana celular y la osmolaridad que genera será efectiva. En cambio, un soluto con un coeficiente de reflexión menor que 1 y que, por ende, permea a través de las membranas, podrá atravesar las membranas y secundariamente determinar también el pasaje de agua. Este último soluto no es osmóticamente activo y la osmolaridad que genera no será efectiva.

A la luz de los conceptos inclusores erróneos frecuentes, el uso de una solución saturada y, por tanto, altamente concentrada de determinado soluto nos llevaría a esperar encontrar plasmólisis en todos los casos; sin embargo, en algunos encontramos turgencia.

Cabe destacar que se trata de protocolos simples y fáciles de poner en práctica en laboratorios de instituciones educativas. Esto permite, además, a partir de preparados microscópicos, hacer inferencias sobre mecanismos moleculares y vincular diversos niveles de organización biológica, aspecto que consideramos de gran relevancia en la enseñanza de las ciencias biológicas.

Para finalizar, enfatizamos la relevancia de:

- Revisar protocolos prácticos antes de su realización, procurando evitar la construcción de conceptos inclusores erróneos, a veces por el uso de protocolos simplificados que no solo no consideran variados factores que influyen en el proceso biológico en estudio, sino que además dejan de lado la diversidad biológica, aspecto central en la enseñanza de las ciencias biológicas.

- Diseñar protocolos prácticos que permitan deconstruir conceptos inclusores erróneos, así como construir en forma consciente conceptos sostén relevantes, para un aprendizaje sustentable.

\section{Referencias}

Aiziczon, B., y Cudmani, L. (2012). Evaluación de una propuesta didáctica en el aula de biofísica de enfermería universitária. Caderno Brasileiro de Ensino de Física, 29(1), 7-32. https://periodicos.ufsc.br/ index.php/fisica/article/view/24277.

Aragüés, A., y Sáez, M. J. (2014). Análisis de una experiencia con maestros en formación: modelo de reacción química y ósmosis. xxvi Encuentros de Didáctica de las Ciencias Experimentales (pp. 277-284). https://bit.ly/2nPgxwA

Burbano Rosero, E. M., Caetano de Almeida, B., Otero, I. y Álvarez, S. (2017). Manual de biología molecular: procedimientos básicos. http://sired.udenar. edu.co/4696/1/ManualdeBiologíaCelular.pdf.

Camarena Rosales, F. (2017). Biología celulary molecular. Manual de prácticas 2017-2. Facultad de Ciencias. Universidad Autónoma de Baja California. http://peces.ens.uabc.mx/bcym/practica/ 2017-2-manual\%20BCYM.PDF.

Chávez Torres, J., Medina Barriga, M., Sánchez Suárez, S. y Mendoza Olivares, R. (2013). Manual del Laboratorio de Biología Celular y Molecular I. Facultad de Biología. Universidad Michoacana de San Nicolás de Hidalgo. https://anacanas.files.wordpress. com/2015/08/manual_del_lab_de_biol_cel_y_ molec_i_2013.pdf.

Cingolani, H. E. y Houssay, A. B. (2006). Fisiología humana (7. ${ }^{a}$ edición). Buenos Aires: El Ateneo. Contreras Ramírez, N. (2017). Manual de prácticas de laboratorio de fisiología del ciclo escolar 2016-2017. Departamento de Fisiología. Facultad de Medicina. Universidad Nacional Autónoma de México. http://www.facmed.unam.mx/fm/pa/2010/ practicas/practicas_fisiologia.pdf

Corominas, J. (2010). Patatas y huevos osmóticos. Revista Eureka Sobre Enseñanza Y Divulgación De Las Ciencias, 7(1), 151-157. https://revistas.uca.es/ index.php/eureka/article/view/2635 
Díaz González, R. y Abuín Figueras, G. (1988). Contribución al estudio de las dificultades en la comprensión del fenómeno osmótico. VI Jornadas de Estudio sobre la Investigación en la Escuela (pp. 21-25). https://idus.us.es/xmlui/ bitstream/handle/11441/49889/EPyLEC_3. pdf? sequence $=1$ \&isAllowed $=\mathrm{y}$.

Frumento, A. S. (1995). Biofísica. Madrid: Mosby/Doyma Libros.

Galagovsky, L. (2004a). Del aprendizaje significativo al aprendizaje sustentable. Parte 1: el modelo teórico. Enseñanza de las Ciencias, 22(2), 229-240. https://ddd.uab.cat/record/1657? ln=ca.

Galagovsky, L. (2004b). Del aprendizaje significativo al aprendizaje sustentable. Parte 2: derivaciones comunicacionales y didácticas. Enseñanza de las Ciencias, 22(3), 349-364. https://core.ac.uk/download/pdf/38990142.pdf

Glaser, R. (2003). Biofísica. Zaragoza, España: Acribia.

Gómez, D. T. Z. e Illana, D. V. O. (2007). Laboratorio de Biología celular. Licenciatura en Biofísica. Facultad de Ciencias. Universidad Autónoma de San Luis de Potosí. http://www.fc.uas/p.mx/informacion-para/ material-didactico/ManualdePracticasparaelLaboratoriodeBilogiaCelular.pdf

Khan Academy (s.f.) https://es.khanacademy.org/ science/biology/membranes-and-transport/ diffusion-and-osmosis/a/osmosis

Latorre, R., López Barneo, J., Bezanilla, F. y Llinás, R. (eds.) (1996). Biofísica y fisiología celular. Universidad de Sevilla. Secretariado de Publicaciones.

López Larrama, M. N., Tucci Añón, J. y Píriz Giménez, N. (2016). Ósmosis: máscara frecuente del transporte de agua en los seres vivos. Una propuesta práctica y su análisis didáctico. Ponencia presentada en las XII Jornadas Nacionales y vil Congreso Internacional de Enseñanza de la Biología. Buenos Aires, Argentina.
Píriz Giménez, N. (2016). Biofísica para la formación del

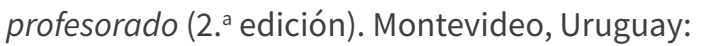
Ediciones Ciencia. Libro bajo licencia cc. https://bit.ly/2pjAJah

Píriz Giménez, N. y López Larrama, M. N. (2011). Células sometidas a diversas soluciones: una vieja actividad práctica desde un análisis teórico. Propuesta de una ampliación. En M. Saravay, S. Umpierrez Oroño y S. Vieira (comps.), Actividades prácticas de biología en la formación de docentes: una sistematización reflexiva (pp. 31-36). Departamento de Ciencias Biológicas, Consejo de Formación en Educación. http://www.cfe.edu.uy/index. php/publicaciones/departamento-de-bilogia.

Píriz Giménez, N., Tucci, J. y Cantero, J. (2018). Cuando las actividades prácticas refuerzan conceptos inclusores erróneos. El caso del transporte de agua. Ponencia presentada durante las XIII Jornadas Nacionales-VIII Congreso Internacional de Enseñanza de la Biología, el vi Seminario Iberoamericano CTS y el x Seminario CTS. Universidad de Quilmes, Buenos Aires, Argentina.

Ramos Medina, E. M. (2016). Propuesta metodológica para la enseñanza de los mecanismos de transporte celular a estudiantes de sexto grado (tesis de doctorado). Universidad Nacional de Colombia. http://www.bdigital.unal.edu.co/54097/.

Torre Casares, J. (coord.) (2005). Prácticas de Biología y Ecología. Consejería de Educación y Ciencia. Centro del Profesorado y de Recursos de Nor-Occidente. http://www.iesdeluarca.es/files/PDFS/LIBRO.PDF. 